\title{
Unsicherheit bewältigen, Gewalt verhindern, Netzwerk bilden
}

\section{Blickdiagnosen}

\section{Welche Diagnose vermuten Sie?}

Ein 65-jähriger Mann wurde 2008 wegen leichter Dyspnoe an unsere Abteilung überwiesen. Der Patient hatte nie geraucht und wies keine Asbestexposition in der Vorgeschichte auf. 2006 hatte er eine Episode mit Fieber, Polyarthralgie und Erythem der Finger, welche spontan wieder abklang; Thoraxröntgenbilder aus dieser Zeit (Abb. 1) zeigen eine dichte Verschat-

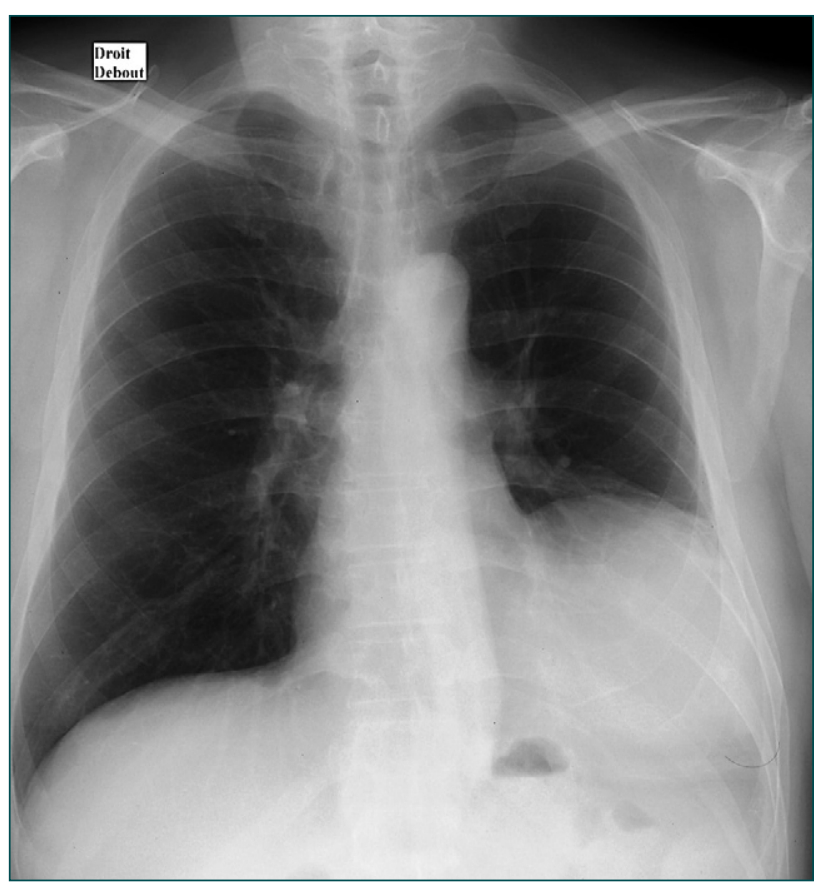

Abb. 1. Der 2006 aufgenommene Röntgenthorax zeigt eine Raumforderung im linken Hemithorax. tung der linken Basis, die als infektiöse Pneumonie interpretiert wurde; unter Antibiotikatherapie trat klinische Besserung ein; danach brach der Kontakt zum Patienten ab. In der Folge stellte er sich jedoch erneut mit chronischer Arthralgie, leichtem Fieber, Nachtschweiß und Abgeschlagenheit vor; diese Beschwerden zeigten von 2006 bis 2008 progressive Verschlechterung. Ein Rheumatologe zog die Diagnose einer rheumatoiden Arthritis in Betracht.

Nach Überweisung an unser Zentrum ergab die klinische Untersuchung eine erythematöse Trommelschlägelbildung der Finger- (Abb. 2) und Zehenendglieder sowie eine Dämpfung im linken Hemithorax. Im Thoraxröntgen zeigte sich links basal eine Verschattung (Abb. 3), die im Vergleich zu 2006 an Größe zugenommen hatte. Ein Thorax-CT ergab außerdem eine Raumforderung von $15 \times 11 \mathrm{~cm}$ Durchmesser, die im basalen Anteil des linken Hemithorax lokalisiert war. Mediastinale Lymphadenopathie war nicht zu erkennen. Unter Kontrastmittelverstärkung zeigte die Raumforderung heterogene Densität bei regelmäßiger Form, ohne Luftbronchogramm oder Invasion benachbarter Strukturen. Eine Fiberbronchoskopie ergab eine extrinsische Stenose des linken Unterlappen bronchus. Biopsien der Bronchien blieben ohne Befund. Eine Knochenszintigraphie ergab ein typisches Muster von periostaler Hyperfixation, das auf eine paraneoplastische hypertrophe pulmonale Osteoarthropathie hindeutet (Abb. 2). 

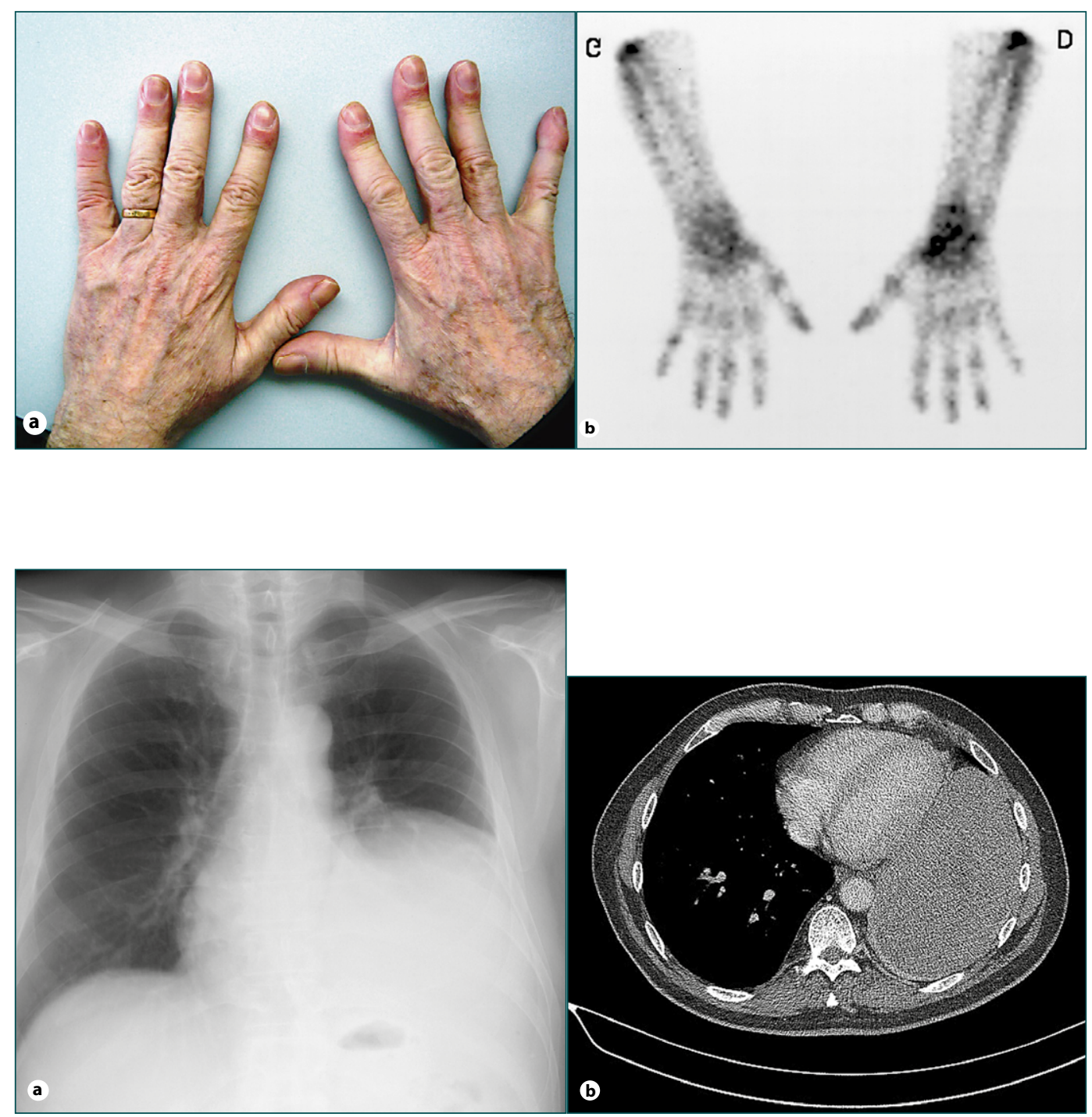

Wie lautet Ihre Diagnose?

\section{Auflösung unter:}

www.karger.com/blickdiagnose-kkp-6-2020

Übersetzung aus Respiration. 2012;84:150-154; DOI: 10.1159/000338115

Kontakt: Vincent Cottin, Hôpital Louis Pradel, 69677 Lyon Cedex, Frankreich, vincent.cottin@chu-lyon.fr
Abb. 2. Trommelschlägelfinger bei der Erstvorstellung. (a) Makroskopisches Erscheinungsbild. (b) Tc99m-Knochenszintigraphie bei der Erstvorstellung.

Abb. 3. Thoraxbildgebung bei der Vorstellung 2008. (a) Im Röntgenbild ist die Größenzunahme der Raumforderung im linken Hemithorax zu erkennen (b) Das Thorax-CT zeigt eine umfangreiche, homogene solide Raumforderung im unteren linken Hemithorax. Pleurale Anomalien im Sinne einer Invasion der sind nicht zu erkennen. 


\section{Medizinstudium}

\section{Das Medizinstudium, das Praktische Jahr und die COVID-19-Pandemie}

Auch wenn das Thema COVID-19 mittlerweile in vielen Facetten in der Öffentlichkeit besprochen wurde, ist der Alltag von Medizinstudierenden noch eher wenig thematisiert worden. Die COVID19-Pandemie, die spätestens seit März 2020 den Klinikalltag in nahezu allen Fachrichtungen beeinflusst, hatte von Beginn an starke Auswirkungen auf den Studienverlauf. Die erste Welle der Pandemie traf auf die Medizinstudierenden, als sich diese in den Semesterferien in Famulaturen und Praktika oder mitten in der Vorbereitung auf die Staatsexamina (M1, M2, M3) befanden.

Wir von der Bundesvertretung der Medizinstudierenden in Deutschland (bvmd e.V.) erhielten in dieser Zeit beispielsweise viele Nachrichten von Kommilitoninnen und Kommilitonen, deren Famulaturen plötzlich abgebrochen wurden. Es herrschte zu Beginn viel Unsicherheit in den Kliniken und Praxen, wie mit dieser besonderen Situation umzugehen sei. Dies war der Einmaligkeit der Situation geschuldet, auf die die Politik in Bezug auf das Studium nicht vorbereitet war. So mussten Mitte März die Staatsexamina-Prüflinge feststellen, dass ihre Prüfungstermine in Gefahr waren. Und nicht nur das: Die verschiedenen zuständigen Ministerien auf Bundes- und Länderebene waren sich nicht einig, wie gehandelt werden solle [1].

Die bvmd e.V. stellte in dieser Situation, in der von Politik und medizinischen Fakultäten aufgrund der hohen Dynamik der Entwicklungen schnell gehandelt werden musste, klar, dass «das lokale Vorgehen in den Ländern in engem Rahmen national abzustimmen und zu synchronisieren» sei [2].

Am 30.03.2020 entschied das Bundesministerium für Gesundheit (BMG) schließlich mittels einer Abweichungsverordnung trotz der national einheitlichen Prüfungsinhalte und dem zu dieser Zeit heterogenen Infektionsgeschehen, dass jedes Bundesland einzeln entscheiden solle, ob die Prüfung stattfindet oder ins kommende Jahr verschoben wird [3].

In 14 Bundesländern durfte die Prüfung dann abgelegt werden, während die Prüflinge in Bayern und Baden-Württemberg unmittelbar ins Praktische Jahr (PJ) starten mussten und nächstes Jahr ein «Hammerexamen» (schriftlich und mündlich) ablegen werden. Der durch diese Abweichungsverordnung entstandene Flickenteppich, die Kurzfristigkeit der Entscheidung wenige Tage vor der Prüfung und die Einschränkungen im PJ (s.u.) sorgte bei vielen Studierenden für große Frustration. Eine durchgeführte Online-Petition fand binnen kurzer Zeit über 100.000 Unterstützerinnen und Unterstützer, die «faire Bedingungen» auch in der Krise forderten [1].

Das PJ nimmt eine besondere Stellung im Medizinstudium ein, da es als vollständig in der Versorgung absolviertes letztes Studienjahr die Schnittstelle zwischen Studium und Praxis darstellt. Möglichst breite Einblicke in die verschiedenen Disziplinen und Abläufe, die schrittweise Übernahme von Verantwortung für eigene Patienten (unter Supervision) sowie das Erlernen zentraler praktischer Fertigkeiten sind für die Vorbereitung des eigenen Berufslebens unabdingbar. Durch die COVID-19-Pandemie ergaben sich sowohl in Organisation als auch in der konkreten Durchführung starke Verände- rungen: Wesentlich ist hierbei die große Einschränkung der PJ-Mobilität zu nennen. Viele PJ-Studierende absolvieren ein oder mehrere Tertiale im Ausland, um weitere Gesundheitssysteme und Kulturen kennenzulernen und Einblicke in ein mögliches Berufsleben (v.a. in der Schweiz) zu erhalten. Für die Wahl des Ortes und Krankenhauses für den Berufsbeginn ist aber auch die innerdeutsche Mobilität essenziell. Durch den bereits erwähnten asynchronen PJ-Beginn in Bayern und Baden-Württemberg, die pandemiebedingten Einschränkungen im Frühjahr und die zahlreichen Risikogebiete im Ausland mussten viele Studierende kurzfristig, oft bereits nach gebuchten Flügen, gemieteten Wohnungen usw., PJPlätze an ihren Heimatuniversitäten belegen.

Auch die Aufgaben im PJ waren - wie auch für die ärztlichen Kollegen - durch die Pandemie stark verändert: Während an einigen Kliniken die Unterstützung in der Bewältigung der Pandemie im Vordergrund stand, und auch Tätigkeiten wie Kontaktnachverfolgung oder Aushilfe bei der Entnahme von Abstrichen im Mittelpunkt standen, wurde uns im Bekanntenkreis auch von Kliniken und Abteilungen (insbesondere chirurgische Bereiche) berichtet, in denen es durch die plötzlich deutlich höhere Zahl an PJ-Studierenden bei gleichzeitig weniger elektiven Operationen zu Leerlauf und geringerem Lerneffekt kam. Ein für die Studierenden positiver Effekt war, dass die PJ-Aufwandsentschädigung an einigen Universitäten angehoben oder erstmalig gewährt wurde, auch wenn dies zum Teil nur temporär Bestand hatte.

Doch Krisen können natürlich auch Chancen sein: Neben dem umfassenden Ausbau digitaler Lehre und der Etablierung neuer Lernformate in allen Semestern des Studiums wurde in der ersten Welle der Pandemie auch die Hilfsbereitschaft der Studierenden deutlich. An allen deutschen Fakultäten meldeten sich viele hunderte Freiwillige, die sich als Hilfskräfte in den Kliniken einbringen wollten. Im Rahmen des \#WIRVSVIRUS-Hackathons der Bundesregierung entstand daraus im Umfeld der Bundesvertretung der Medizinstudierenden in Zusammenarbeit mit der Initiative «Medis vs. COVID19» eine digitale Plattform namens «Match4Healthcare». Dieses in kürzester Zeit entwickelte und programmierte Portal ermöglicht die Kontaktvermittlung zwischen hilfsbereiten Studierenden und einzelnen Kliniken für einen bedarfsgerechten und schnellen Einsatz. Ein gelungenes Beispiel einer relevanten digitalen Anwendung ist entstanden, die Mitte 2020 durch die «Zusammen gegen Corona»-Initiative des BMG ausgezeichnet wurde [1].

Als Fazit kann festgehalten werden, dass die Pandemie eine große Herausforderung nicht nur für die Krankenhäuser, sondern auch für Studierende und Fakultäten darstellte. Wir sind zuversichtlich, dass die Hilfsbereitschaft und Flexibilität der Medizinstudierenden auch in den kommenden Monaten anhalten wird und die Bekämpfung der Pandemie gleichzeitig, auch durch nun etablierte digitale Methoden, etwas weniger stark Lehre und Prüfungen beeinflussen wird. Trotzdem darf auch gerade vor dem Hintergrund eines vermutlich noch mehrere Monate bestehenden Ausnahmezustands nicht vergessen werden, dass die Studierenden von heute die Ärz-
336

Kompass Pneumol 2020;8:334-340 DOI: $10.1159 / 000512301$ 
teschaft von morgen darstellen und deshalb in Lehre und Wertschätzung auch in Pandemiezeiten in unser aller Interesse Einschränkungen auf das Nötigste reduziert werden sollten.

\section{Literatur}

1 Kaufmann MD: Auswirkungen auf das Medizinstudium durch COVID-19. Kompass Ophthalmol. 2020;6:142-146. Bei dem nun vorliegenden Text handelt es sich um eine aktualisierte und erweiterte Fassung.

2 Bundesvertretung der Medizinstudierenden in Deutschland e.V., Stellungnahme «Empfehlungen der bvmd zum Umgang mit der COVID-19-Pandemie an den Medizinischen Fakultäten und in der Versorgung», 16.03.2020.

3 Bundesministerium für Gesundheit (BMG), Verordnung zur Abweichung von der Approbationsordnung für Ärzte bei einer epidemischen Lage von nationaler Tragweite, 30.03.2020.
Autoren: Matthias D. Kaufmann, Assistenzarzt am Universitätsklinikum Erlangen seit 2020, 2019/2020 Vizepräsident der Bundesvertretung der Medizinstudierenden in Deutschland (bvmd e.V); Jeremy Schmidt, PJ-Studierender an der Universität Heidelberg, 2019 Bundeskoordinator für medizinische Ausbildung der Bundesvertretung der Medizinstudierenden in Deutschland (bvmd e.V), Mitgründer von match4healthcare e.V.

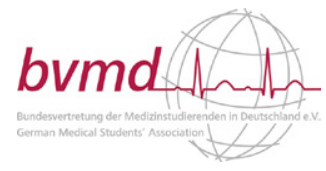

\section{Aus der Praxis}

\section{Kein Opfer werden: So reagierst Du souverän auf gewalttätige Patienten}

Drohungen, Beleidigungen oder sogar Handgreiflichkeiten: Gewalttätige Übergriffe gegen Ärzte nehmen - zumindest gefühlt immer weiter zu. Der Fachmann für Kriminalprävention Dr. Martin Eichhorn gibt Tipps, wie Du im Ernstfall am besten reagieren kannst. In der Notaufnahme verliert ein Patient die Nerven und fängt an, einen Arzt oder das Pflegepersonal zu beschimpfen. Im Wartezimmer wird ein Vater laut, der nicht weiß, wie es seinem kleinen Sohn geht. Im Rettungswagen randaliert ein alkoholisierter Patient und schlägt auf die Rettungskräfte ein. Es sind oft Ausnahmesituationen wie diese, in denen Patienten gewalttätig werden. «Wichtige Faktoren, wenn es zu Gewalt gegen medizinisches Personal kommt, sind oft Schmerzen, Alkohol - aber auch Sorge um Angehörige», verrät Eichhorn, «Aggression entsteht oft aus Unsicherheit». Eine gute Maßnahme zur Prävention sei es, beispielsweise die nächsten Schritte oder die Wartezeiten besser zu kommunizieren.

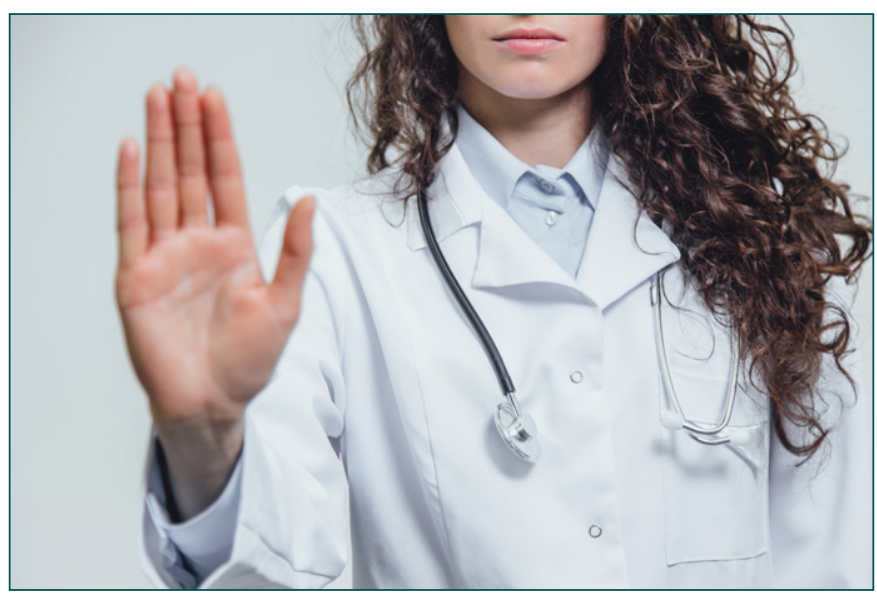

Stop! Wenn ein Patient verbal oder körperlich übergriffig wird, musst Du das nicht einfach hinnehmen. Wer sich ein bisschen vorbereitet, kann sich souverän gegen eine Beleidigung oder einen Angriff wehren.

(c) Andrii Bicher / iStock

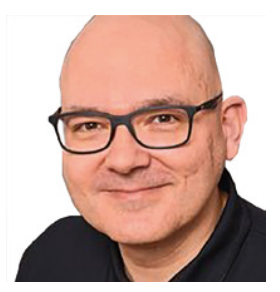

Dr. Martin Eichhorn ist zertifizierte Fachkraft für Kriminalprävention | privat

\section{Die häufigsten Angreifer: Männlich und jünger als 25 Jahre}

«Gewalttäter sind meistens männlich und jünger als 25 Jahre», erklärt der Präventionsexperte. Natürlich gebe es Ausnahmen, aber genau deshalb seien Gynäkologen seltener von Gewalt betroffen als andere Fachärzte. Stark betroffen seien dagegen Notärzte, Rettungskräfte und alle, die in der Notaufnahme arbeiten - aber Konflikte mit Patienten könne es grundsätzlich in allen Bereichen geben. Oft spielt auch Stress und Personalmangel auf ärztlicher Seite eine Rolle: «Nach einer langen Schicht reagiert man oft nicht mehr so entspannt und deeskalierend, wie es wünschenswert wäre. Aber trotzdem würde ich sagen, dass der größte Teil der Konflikte von den Patienten ausgeht», schätzt Eichhorn.

Grundsätzlich muss zwischen verbaler Gewalt - also Drohungen, Beschimpfungen, Beleidigungen - und körperlicher Gewalt unterschieden werden. «Verbale Übergriffe sind natürlich deutlich häufiger als körperliche Gewalt», erklärt der Experte. Je nach Herkunft und Milieu sind es aber die meisten Mediziner nicht gewohnt, mit verbalen Übergriffen umzugehen: «Die meisten sind erstmal verschreckt, überfordert und verstummen unter Umständen. Schlagfertigkeit wäre gut - aber das ist in so einer Situation nur den wenigsten gegeben».

\section{«Ich helfe Ihnen gern, aber nicht in diesem Ton»}

Eichhorn empfiehlt daher eine gute Vorbereitung auf mögliche verbale Angriffe: Sein Rat ist es, sich schon im Vorfeld ein paar Formulierungen zurechtzulegen, mit denen man sich wohl fühlt und 
die zu einem passen. «Im Grunde reicht schon eine ganz minimale Reaktion», verrät der Präventionsexperte. Also: Gelassen bleiben und mit «so, so» oder «ach was» auf die Beleidigung reagieren - das nimmt dem Aggressor den Wind aus den Segeln. «Man kann auch sagen «lch helfe Ihnen gern, aber nicht in diesem Ton» und dem anderen so eine Grenze aufzeigen. Oder wenn man etwas schlagfertiger wirken möchte, kann man auch fragen: «Oh, können wir diesen Teil überspringen?> oder «Gibt es Sie auch in nett?». Mit dem richtigen Spruch im richtigen Moment kann man Leute mundtot machen», rät Eichhorn. Es gehe vor allem darum, zu zeigen, dass man nicht als Opfer bereitstehe.

Und auch bei körperlicher Gewalt reagieren die meisten Menschen erstmal überfordert. Spontan neigen wir seit Urzeiten zu drei Verhaltensmustern, wenn wir angegriffen werden:

- Flucht: Wenn wir weglaufen können, laufen wir weg.

- Gegenangriff: Wenn wir nicht weglaufen können oder es eher unserer Persönlichkeit entspricht, setzen wir uns zur Wehr und kämpfen.

- Schockstarre: Das Gewaltopfer friert quasi ein und bewegt sich überhaupt nicht mehr. «Das hat unseren Vorfahren geholfen, weil viele tierische Fressfeinde auf Bewegung reagieren. Heute hilft es uns leider nicht mehr», erklärt Eichhorn.

\section{Sich effektiv wehren}

Das Problem: Der Angreifer rechnet bei seinen Opfern auch mit diesen Verhaltensmustern - und meistens ist er seinem Opfer überlegen. Sei es, weil er in seiner Biografie schon viel Erfahrung mit Gewalt gesammelt hat, sei es, weil er körperlich kräftiger ist, oder weil er die Überraschung auf seiner Seite hat. «Das Opfer sollte etwas tun, was nicht in dieses Schema fällt - das überfordert und verwirrt den Gewalttäter und gibt dem Opfer die Möglichkeit, sich aus der Affäre zu ziehen», rät Eichhorn. Dazu empfiehlt er Präventionsexperte drei Methoden:

1 Angreifer abwehren durch Lärm: Wenn Du den Angreifer mit ganzer Kraft anbrüllst, kannst Du ihn tatsächlich in die Flucht schlagen. Positiver Nebeneffekt: Das Geschrei macht andere Menschen in der Umgebung wie Kollegen oder andere Patienten auf Deine Notlage aufmerksam. Denn Öffentlichkeit ist immer wichtig: So werden andere Leute zu Zeugen und können eventuell weitere Hilfe rufen. Alternativ zur eigenen Stimme kann auch ein Taschenalarm helfen, der einen Ton von 110 Dezibel auslösen kann.

2. Angreifer abwehren durch Ekel: Eine zweite und weniger bekannte Methode ist es, den Angreifer durch Ekelgefühle abzuschrecken. «Ekel setzt den gesamten Organismus in Alarmbereitschaft - wenn etwas Ekliges auf der Straße liegt, schreckt man sofort zurück», erklärt Eichhorn. Konkret auf den Angreifer bezogen bedeutet das: Wenn Du plötzlich kräftig hustest und würgst und so tust, als würdest Du Dich gleich übergeben, löst das ein Ekel-Kopfkino beim Angreifer aus. Alternativ hilft es auch, etwas von «Durchfall, die ganze Nacht, lassen Sie mich durch, ich muss aufs Klo» zu murmeln. Auch das bringt den Angreifer auf Distanz und kann verhindern, dass er zuschlägt.
3. Angreifer abwehren durch simulierte Krankheit: Egal, ob Du einen Herzinfarkt, einen epileptischen Anfall oder plötzliche Blindheit simulierst: All das kann dafür sorgen, den Gewalttäter zu verwirren und von seinem Angriff abzuhalten. Nur glaubwürdig muss es sein - zumindest für einen Laien. Als Mediziner bist Du hier im Vorteil, weil Du täglich mit Krankheiten zu tun hast und die Symptome kennst. Wichtig: Auf keinen Fall beim Schauspielern zu Boden gehen - dann bist Du dem Angreifer gegenüber in einer schwächeren Position.

Bei allen Methoden gilt: Sie halten den Angreifer nicht dauerhaft auf - aber sie können Dir Zeit geben, zu flüchten und Dich in Sicherheit zu bringen. Und: Das klappt wahrscheinlich alles nicht spontan. Du solltest Dir also regelmäßig etwa einmal pro Monat Zeit nehmen, das Szenario und Deine mögliche Reaktion in Gedanken durchzuspielen.

\section{Die Polizei, Dein Freund und Helfer}

Und wann sollten wir die Polizei rufen? «Nach meiner Erfahrung wird die Polizei im medizinischen Bereich eher zu selten gerufen», verrät Eichhorn. Mediziner sollten sich klar machen, dass die Polizei eine Dienstleisterin sei, deren Aufgabe es sei, genau solche Probleme zu lösen. «Gerade Menschen in helfenden Berufen haben aber eine unglaublich hohe Schmerzgrenze, wenn es darum geht, die Polizei hinzuzuziehen», beklagt der Präventionsexperte. «Grundsätzlich würde ich empfehlen, sich nicht so viel bieten zu lassen und die Polizei eher zu rufen bzw. zu kontaktieren - auch bei Beleidigungen oder sexueller Belästigung».

Dr. Martin Eichhorn ist zertifizierte Fachkraft für Kriminalprävention und zertifizierter Trainer (TU Berlin). Er bietet im gesamten deutschsprachigen Raum Inhouse-Seminare zu Themen wie «Sicheres Handeln in eskalierenden Konflikten», «Deeskalation» oder «Umgang mit Grenzüberschreitungen und Beleidigungen» an.

Unter anderem hat er für die Ärztekammer Niedersachsen eine Broschüre verfasst, die viele hilfreiche Tipps zum Thema Gewaltprävention aufgreift: «Übergriffe gegen Praxisteams - Vorbeugen und abwenden» (www.aekn.de/fileadmin/media/Down loadcenter/gewalt_gegen_aerzte/brosch-uebergriffe-praxis_web. pdf)

Mehr Infos: www.martin-eichhorn.berlin

Quelle: Kein Opfer werden: So reagierst Du souverän auf gewalttätige Patienten. Ein Beitrag auf www.operation-karriere.de (www. operation-karriere.de/karriereweg/assistenzarzt/kein-opfer-werden -so-reagierst-du-souveraen-auf-gewalttaetige-patienten.html) DOI: $10.1159 / 000512301$ 
Junge Ärztinnen und Ärzte

Vernetzen. Mitwirken. Bilden.

- Nachwuchsförderung in der Hämatologie und Onkologie durch den Arbeitskreis Junge DGHO

\section{Hintergrund}

Kaum ein anderes medizinisches Fachgebiet erfährt eine solch rasante Entwicklung wie die Hämatologie und Medizinische Onkologie. Angesichts der stetig wachsenden Zahl maligner Tumorerkrankungen in einer alternden Bevölkerung wird dieses Fach auch in Zukunft eine zentrale Rolle in der internistischen Patientenversorgung spielen. Das beständig zunehmende Verständnis der Biologie maligner Tumorerkrankungen, die Etablierung immer neuer molekular zielgerichteter und zellbasierter Therapieoptionen und eine bessere Informiertheit der Patienten stellen in der Hämatologie und Onkologie tätige junge Ärztinnen und Ärzte täglich vor die Herausforderung mit diesen Entwicklungen Schritt zu halten um den an Sie gestellten Ansprüchen gerecht zu werden und die bestmögliche Patientenversorgung sicherzustellen [1].

\section{Der Arbeitskreis Junge DGHO}

Der Arbeitskreis Junge DGHO wurde auf der Jahrestagung der Deutschen Gesellschaft für Hämatologie und Onkologie, der Österreichischen Gesellschaft für Hämatologie und Onkologie (OeGHO) und der Schweizerischen Fachgesellschaften für Hämatologie (SGH) und Medizinische Onkologie (SSMO) im Herbst 2018 in Wien gegründet. Der Arbeitskreis hat aktuell 17 Mitglieder aus verschiedensten Krankenversorgungseinrichtungen der gesamten Bundesrepublik, die sich (abgesehen von Zeiten der COVID-19-Pandemie) zweimal jährlich im Rahmen der DGHO-Kongresse im Frühjahr und Herbst treffen. Zentrale Themen für die Arbeitskreisaktivitäten sind u.a.:

1. Sicherung der Qualität der Facharztweiterbildung,

2. Aufklärung über die verschiedenen Karriereoptionen für junge Hämatologinnen und Hämatologen/Medizinische Onkologinnen und Onkologen,

Wichtigste Anliegen des Arbeitskreises Junge DGHO

- Sicherung der Zukunftsfähigkeit und Attraktivität der Hämatologie und Onkologie am Standort Deutschland.

- Institutionsübergreifende personelle Aufstellung im Arbeitskreis (universitär/kommunal/niedergelassen).

- Interessenvertretung junger Kolleginnen und Kollegen in der Fachgesellschaft zur Mitgestaltung der Zukunftsfähigkeit und Attraktivität unseres vielfältigen und dynamischen Fachgebiets durch Unterstützung und Mitgestaltung der vielfältigen Aktivitäten der $\mathrm{DGHO}$

- Nationale und internationale Vernetzung mit jungen Vertretern anderer hämatologischer/onkologischer, internistischer und mit der Tumormedizin assoziierter Fachgesellschaften.

- Fortbildungsangebote für Onkologie-interessierte Studierende und angehende Hämatologinnen/Hämatologen und Onkologinnen/Onkologen.
3. Vereinbarkeit von Familie und Beruf/Work-Life-Balance und moderne Arbeitszeitmodelle,

4. Verbesserung von Karrierechancen für Ärztinnen und Ärzte in der Hämatologie und Medizinischen Onkologie,

5. Aufwertung ärztlicher Forschungstätigkeit (z.B. Clinician Scientist-Programme) sowie

6. Ausbau der Nachwuchsförderung für interessierte Medizinstudierende.

Der Arbeitskreis soll ein Netzwerk zur Förderung des Austausches zu Ausbildung und Karriereoptionen für junge Hämatologinnen und Hämatologen sowie Medizinische Onkologinnen und Onkologen sein, neue junge Kolleginnen und Kollegen für eine Mitgliedschaft in der DGHO gewinnen, zur aktiven Mitarbeit motivieren und darüber hinaus die gesundheitspolitischen und berufsgruppenspezifischen Interessen junger Kolleginnen und Kollegen formulieren und sie innerhalb der DGHO und nach außen vertreten [1]. Der Arbeitskreis mit seinen Mitgliedern und Projektideen profitiert von engem Kontakt zu den Leitungsgremien der DGHO und nicht zuletzt von einer professionellen organisatorischen Unterstützung durch die DGHO Service $\mathrm{GmbH}$.

\section{Aktuelle Projekte}

Ein brandaktuelles Projekt ist der Aufbau eines Mentoringprogramms für junge Kolleginnen und Kollegen am Beginn und kurz vor Ende der Facharztausbildung in der Inneren Medizin mit Schwerpunkt Hämatologie und Onkologie. Das Programm erhält unmittelbare Unterstützung durch den DGHO-Vorstand (Frau Prof. de Wit, Berlin) und wird den jungen Kolleginnen und Kollegen erfahrene Mentoren als Berater und persönliche Ansprechpartner für Karriereplanung und die erfolgreiche Verwirklichung persönlicher Karriereziele zur Seite stellen. Hierfür wird es ein Bewerbungsverfahren geben, um eine bedarfsorientierte Zuordnung von Mentees und Mentoren zu ermöglichen.

Darüber hinaus ist der Arbeitskreis maßgeblich an der Gestaltung der DGHO-Juniorakademie beteiligt. Diese jährlich im Frühjahr stattfindende, mehrtägige Veranstaltung richtet sich gezielt an interessierte Medizinstudierende und Kolleginnen und Kollegen in der frühen Facharztweiterbildung mit einer Kombination aus Fortbildungen zu aktuellen Entwicklungen unseres Fachgebiets durch ausgewiesene Expertinnen und Experten und Informationen über die praktische Tätigkeit in der Hämatologie und Medizinischen Onkologie. Plenarsitzungen und Workshops, aber auch gemeinsame Gespräche mit Ordinarien und Führungskräften im informellen Rahmen und der intensive Erfahrungsaustausch unter den Teilnehmenden stehen im Fokus. Zusätzlich werden wertvolle Tipps zur Karriereförderung gegeben und Berufsperspektiven aufgezeigt [1]. Nähere Informationen zu Veranstaltungszeitpunkt, -ort und Anmeldefristen sind unter https://www.dgho.de/aktuelles/news/ news/2020/dgho-juniorakademie-2021-jetzt-anmelden zu finden. 


\section{Kooperationen und Netzwerk}

Gemeinsam mit den Kolleginnen und Kollegen der österreichischen OeGHO Youngsters und der Schweizerischen Gesellschaft für Hämatologie gestalten wir seit 2018 einen gemeinsamen Track auf den jährlichen Jahrestagungen mit Fortbildungsveranstaltungen und Vorträgen gezielt für den Nachwuchs der Fachgesellschaften zu Themen, die z.B. den klinischen Alltag für Berufsanfänger, Möglichkeiten der Forschungsförderung oder die individuelle Karriereplanung betreffen. Die Veranstaltungen der Jungen DGHO sollen gleichzeitig ein Treffpunkt für junge Kolleginnen und Kollegen sein, der Anonymität großer Kongresse entgegenwirken und eine Plattform zum Networking und ungezwungenen Austausch darstellen, um z.B. gemeinsame Forschungsprojekte, Aus- und Weiterbildungsangelegenheiten andernorts zu besprechen oder Ideen für ein gemeinsames berufspolitisches Engagement zu entwickeln. Mit den Kolleginnen und Kollegen vorgenannter Fachgesellschaften und in Kooperation mit den Young Medical Oncologists der Arbeitsgemeinschaft Internistische Onkologie (AIO YMOs) realisieren wir zudem Umfragen und Forschungsprojekte, wie zuletzt zum Bewusstsein bezüglich der für die gute wissenschaftliche Praxis schadhaften sogenannten Predatory Journals unter in der Hämatologie und Onkologie tätigen Kolleginnen und Kollegen [2].

Ferner stellt unser Arbeitskreis den Vertreter für Deutschland im Young Oncologist Committee der European Society of Medical Oncology (ESMO YOC) und so tragen wir aktiv zu den bedeutsamen Lehr- und Fortbildungsaktivitäten und Unterstützungsangeboten der ESMO für junge Onkologinnen und Onkologen weit über die Grenzen Europas hinaus bei. Informationen hierzu unter https://www.esmo.org/about-esmo/organisational-structure/youngoncologists-committee.

Durch ein zukünftiges Engagement zusammen mit den jungen Vertretern anderer internistischer Fachgesellschaften im Bündnis Junge Internisten (https://junge-internisten.de) beteiligen wir uns an einem fachübergreifenden Netzwerk, um gemeinsam die Anliegen junger Kolleginnen und Kollegen in der Inneren Medizin in Deutschland zu erfassen und bedarfsorientiert zu verbessern.

\section{Mach mit ...}

So entsteht innerhalb und außerhalb des Arbeitskreises ein großes Netzwerk engagierter, motivierter junger Kolleginnen und Kollegen, von denen jede/r einzelne, die Fachgesellschaften und unser gesamtes Fachgebiet profitieren können. Der Arbeitskreis Junge

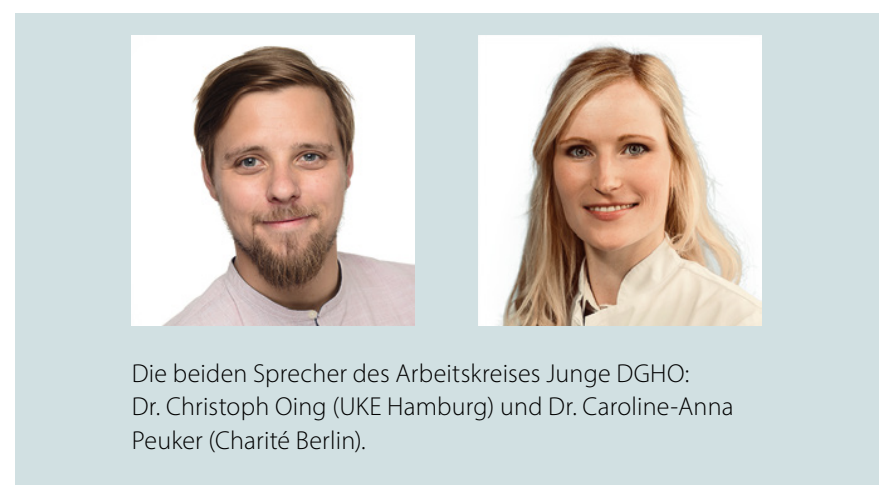

DGHO freut sich über Interesse an einer Mitarbeit in der DGHO und unserem Arbeitskreis. Eine DGHO-Mitgliedschaft bietet neben dem Zugang zum Arbeitskreis viele weitere Vorteile für den Arbeitsalltag durch vollen Zugriff auf alle Inhalte der OnkopediaLeitlinien, relevante Fachinformationen und Neuerungen, das Netzwerk der Fachgesellschaft und vergünstigte Teilnahmegebühren für Tagungen und Fortbildungen.

Kontakt: Nähere Informationen zur Jungen DGHO finden Sie unter https://www.dgho.de/d-g-h-o/junge-dgho oder schreiben Sie an junge-dgho@dgho.de.

\section{Literatur \\ 1 Oing C et al.: FORUM. 2018;33:318-321. \\ 2 Richtig G et al.: ESMO Open. 2019;4(6):e000580.}

Autoren: Dr. Christoph Oing, II. Medizinische Klinik \& Poliklinik, Universitätsklinikum Eppendorf, Hamburg, Mildred Scheel Nachwuchszentrum HaTriCs4, Universitätsklinikum Eppendorf, Hamburg, Martinistr. 52, 20246 Hamburg, Deutschland, junge-dgho@ dgho.de; Dr. med. Caroline Anna Peuker, Charité - Universitätsmedizin Berlin, Klinik für Hämatologie, Onkologie und Tumorimmunologie, Campus Benjamin Franklin, Hindenburgdamm 30, 12200 Berlin

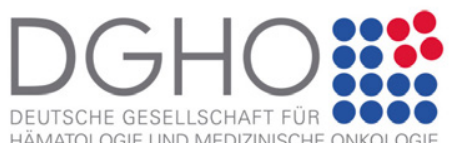

